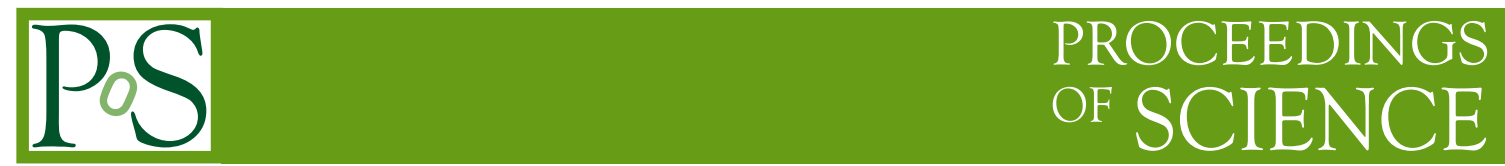

\title{
Searches for new physics in events with multiple leptons with the ATLAS detector
}

\section{Daniel Hayden* on behalf of the ATLAS Collaboration ${ }^{\dagger}$}

Michigan State University

E-mail: danhaydeno@gmail.com

Events containing multiple leptons are useful probes of new phenomena due to the low background from Standard Model processes. This paper describes searches for events with three or more leptons, as well as a search for excited leptons, using data recorded in 2012 at $\sqrt{s}=8 \mathrm{TeV}$, by the ATLAS experiment at the LHC.

The European Physical Society Conference on High Energy Physics

18-24 July, 2013

Stockholm, Sweden

\footnotetext{
*Speaker.

${ }^{\dagger}$ Thank you to all of the ATLAS analysis groups involved in producing these results and contributing material.
} 


\section{Introduction}

There are many searches for physics beyond the Standard Model (SM) conducted at the Large Hadron Collider (LHC) situated at CERN in Geneva, Switzerland. The ATLAS detector [1] is one of four main detectors, built at designated points around the $27 \mathrm{~km}$ accelerator tunnel, to observe proton-proton (pp) collisions at $\sqrt{s}=8 \mathrm{TeV}$ (eventually up to a maximum of $14 \mathrm{TeV}$ ).

The ATLAS detector excels at identifying leptons and photons consisting of subdetectors such as the inner detector tracking systems that allow the tracks of charged particles to be precisely measured. The design of the hermetic and finely segmented electromagnetic calorimeter is able to contain highly energetic electron and photon showers while at the same time maintaining excellent energy resolution, which is further complemented by the extended muon spectrometer systems.

For this reason, searches for new physics in multi-lepton final states are a very promising area of research, especially due to the relatively low expected background from SM processes.

The $\sqrt{s}=8 \mathrm{TeV}$ multiple lepton searches described here pertain to events with three or more leptons (or two leptons and a photon), which comprise three ATLAS analyses:

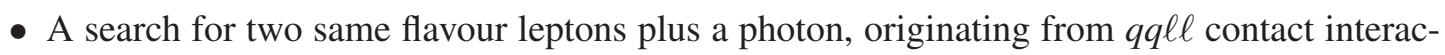
tions with an excited lepton. This analysis uses $13 \mathrm{fb}^{-1}$ of collected data [2].

- A search for three leptons, allowing any combination of electrons and muons as well as up to one tau. This analysis is conducted in a fiducial region in order to achieve a model independent search, and uses the entire 2012 dataset of $20.3 \mathrm{fb}^{-1}$ [3].

- A search for four leptons, resulting from the presence of heavy leptons in the Type III Seesaw model. This analysis uses $5.8 \mathrm{fb}^{-1}$ of collected data [4].

\section{Excited Leptons}

Compositeness models address the fundamental questions of a mass hierarchy in the SM and the generational structure of quarks and leptons, by proposing that quarks and leptons are actually composite particles made up of hypothetical elementary particles called preons [5]. In these models the quarks and leptons are the lowest-energy bound states of preons, with quark-lepton interactions becoming visible at the scale of the constituents' binding energy (or compositeness scale), $\Lambda$, via four fermion contact interactions, which can also give rise to excited states. The production of excited leptons $\left(\ell^{* \pm}\right)$ would be theoretically valid for masses below the scale $\Lambda$, and in nearly all models the underlying preon dynamics are ignored. Terms in the gauge-mediated Lagrangian of the well studied homodoublet-type $\ell^{*}[6,7,8]$ allow for the transition of the excited state into the ground state lepton via an electromagnetic radiative mode $\ell^{* \pm} \rightarrow \ell^{ \pm} \gamma$. For a fixed value of $\Lambda$, the branching ratio $\mathrm{B}\left(\ell^{* \pm} \rightarrow \ell^{ \pm} \gamma\right)$ decreases rapidly with increasing $\ell^{*}$ mass, competing with the decay channel $\ell^{* \pm} \rightarrow \ell^{ \pm} f \bar{f}$. Searches at previous experiments such as LEP [9], HERA [10], and the Tevatron [11] have found no evidence for excited leptons. Additionally, previous searches at ATLAS [12] and CMS [13] using $5 \mathrm{fb}^{-1}$ at $\sqrt{s}=7 \mathrm{TeV}$, have excluded $e^{*}$ and $\mu^{*}$ masses below $2 \mathrm{TeV}$ at $95 \%$ confidence level (CL) for the special case where $\Lambda=m_{\ell}^{*}$. 


\subsection{Analysis Strategy and Selection Criteria}

This search focuses on the production of a single excited lepton $\left(e^{*} / \mu^{*}\right)$ in association with a lepton, giving two same-flavour opposite-charge leptons, plus a photon, in the final state $(p p \rightarrow$ $\left.\ell \ell^{*} \rightarrow \ell^{+} \ell^{-} \gamma\right)$. All three particles are typically very energetic, isolated, and well separated from one another, with the event kinematics determined by two unknown parameters $m_{\ell^{*}}$ and $\Lambda$. The analysis performs a search for an excess in the $\ell^{+} \ell^{-} \gamma$ invariant mass $\left(m_{\ell^{+} \ell^{-}} \gamma\right)$ spectrum.

The dominant background to this search arises from the Drell-Yan process ( $p p \rightarrow Z / \gamma^{*} \rightarrow$ $\ell^{+} \ell^{-}$), accompanied by either a prompt photon from initial/final state radiation (irreducible $Z / \gamma^{*}+\gamma$ contribution) or a jet misidentified as a photon (reducible $Z / \gamma^{*}+$ jets contribution). The latter is suppressed by imposing stringent quality requirements on the photon candidate. Contributions from $\mathrm{W}+\mathrm{jets}$ and multijet events are reduced to negligible levels by requiring that leptons and photons be isolated, with small contributions from $t \bar{t}$ and diboson processes in both channels.

The $e^{*}$ search uses events fired by a calorimeter-only trigger requiring two clusters with $p_{T_{\text {lead }}}>35 \mathrm{GeV}$ and $p_{T_{\text {sub }}}>25 \mathrm{GeV}$, respectively. Two electron candidates are required to be within $|\eta|<2.47$ (excluding the crack region $1.37<|\eta|<1.52$ ), and to have $p_{T_{\text {lead }}}>40 \mathrm{GeV}$, $p_{T_{\text {sub }}}>25 \mathrm{GeV}$. Furthermore, electron identification criteria using shower shape variables are applied to ensure good quality candidates. Lastly to reduce background from jets, the leading electron is required to pass an isolation criterion demanding that the $\sum p_{T}$, in a cone of radius $\Delta R=\sqrt{\Delta \eta^{2}+\Delta \phi^{2}}<0.2$ (subtracting the core of the energy deposition), be less than $7 \mathrm{GeV}$. For the $\mu^{*}$ search, a single-muon trigger with matching tracks in the muon spectrometer and inner detector is used, requiring a combined track $p_{T}>25 \mathrm{GeV}$. Two muon candidates are subsequently required to be within $|\eta|<2.5$, have $p_{T}>25 \mathrm{GeV}$, and have opposite charge. Each muon is also required to have a minimum number of hits in the inner detector and each of the inner, middle, and outer layers of the muon spectrometer to optimise the momentum resolution. To reduce contributions from heavy flavour hadrons, each muon is required to be isolated such that $\left(\sum p_{T}^{\text {tracks }}(\Delta \mathrm{R}<0.3)\right) / p_{T}<0.05$. In both searches, if more than one pair passes all of the above criteria, the pair with the largest invariant mass is selected. The presence of at least one photon candidate with $p_{T}>30 \mathrm{GeV}$ and $|\eta|<2.37$ (excluding the crack region) is required in both channels. Photon candidates have to meet stringent photon identification criteria, passing an isolation criterion of less than $10 \mathrm{GeV}$ in a cone of $\Delta R<0.4$ and $\Delta R(\ell, \gamma)>0.7$.

\subsection{Results}

The resulting data/MC invariant mass distribution for $\mathrm{e}^{*}$ is shown in Figure 1, with no apparent signal excess observed in either the electron or muon channel. In the $m_{\ell^{*}}$ range between $0.2 \mathrm{TeV}$ and $2.0 \mathrm{TeV}$, the systematic uncertainties in both channels vary between $6-10 \%$ for signal and 10 $40 \%$ for background estimates. The significance of an excess in data is quantified using a $p$-value, the probability of observing a signal-like fluctuation greater than or equal to the data observation, given that the background-only hypothesis is true. The minimum $p$-value of $16 \%$ is observed in the $e^{*}$ search for $m_{e e \gamma}>1050 \mathrm{GeV}$, with no apparrent excess in the $\mu^{*}$ search.

Given the absence of a significant excess of events, an upper limit is set on the $\ell^{*}$ cross-section times branching ratio $(\sigma \mathrm{B})$ at $95 \% \mathrm{CL}$. The statistical interpretation uses a Bayesian approach [14] with a flat positive prior on $\sigma \mathrm{B}$, and systematic uncertainties incorporated as nuisance parameters 

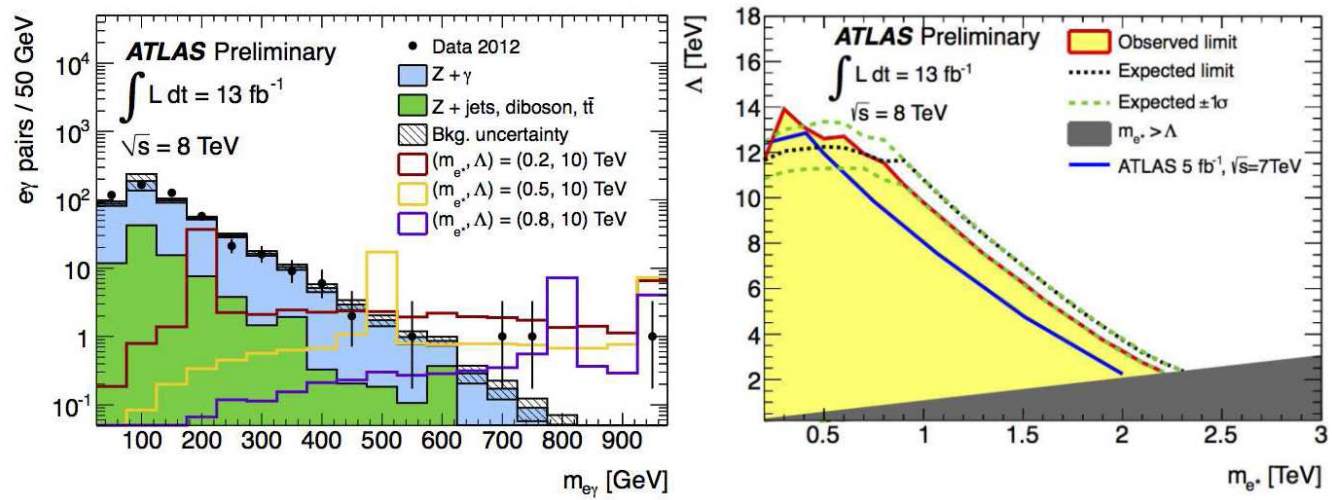

Figure 1: (left) $\ell \gamma$ invariant mass distribution for the $e^{*}$ channel $\left(m_{\ell \ell}>110 \mathrm{GeV}\right)$. The last bin contains the sum of all entries with $m_{\ell \gamma}>925 \mathrm{GeV}$. Signal predictions for $m_{\ell^{*}}$ with $\Lambda=10 \mathrm{TeV}$ are shown. (right) Exclusion limits in the $m_{\ell^{*}} \Lambda$ plane for $e^{*}$. The regions to the left of the limit lines represent areas excluded at 95\% CL, with the dark shaded region denoting $m_{\ell^{*}}>\Lambda$ which is not applicable for the model considered [2].

with Gaussian priors. The limits are translated into bounds on $\Lambda$ as a function of $m_{\ell^{*}}$, using the theoretical dependence of $\sigma \mathrm{B}$ on $\Lambda$. By varying $\Lambda$ it is then possible to evaluate the excluded region on the $m_{\ell^{*}} \Lambda$ plane for this search, which is presented in Figure 1 for the electron channel.

\section{Three Charged Leptons}

Processes containing three charged leptons in their final state are rare in the SM, being dominated by the diboson production of $\mathrm{WZ}$ and $\mathrm{ZZ}$. Therefore any excess of three charged lepton events over the SM prediction would be very interesting.

\subsection{Analysis Strategy and Selection Criteria}

This analysis conducts a model independent search for new physics in the $\ell \ell \ell$ channel, defining various interesting signal regions. The strengths of this search reside in a low lepton threshold, the inclusion of channels with up to one hadronically decaying $\tau$, and the probing of many kinematic variables with signal potential. A nice feature of this search is that per-lepton efficiencies are also provided so that the model independent limits can be interpreted for specfic models.

The search is first divided into four mutually exclusive signal channels, based on the final states containing: $\geq 3 \ell$ (off-Z), $\geq 3 \ell$ (on-Z), $2 \ell+\tau$ (off-Z), and $2 \ell+\tau$ (on-Z). Where "on-Z" and "off-Z" is defined as $|m(\ell \ell)-m(Z)|$ less than or greater than $20 \mathrm{GeV}$, respectively. Additionally, all opposite-charge same-flavour lepton pairs with $m\left(\ell^{+} \ell^{-}\right)<15 \mathrm{GeV}$ are rejected. The lepton selection requires a minimum $p_{T}$ threshold of $15 \mathrm{GeV}$, within a fiducial volume $(|\eta|<2.5)$ and stringent lepton flavour identification. The e/ $\mu$ specific selections also further require calorimeter and track isolation. For each of the four defined channels, there is further division into kinematic regions based on many variables of interest, resulting in a total of 94 signal regions.

\subsection{Results}

The total number of expected and observed events in each of the four channels is presented in Table 1. No clear excess is observed in data above the SM prediction, therefore upper limits are 


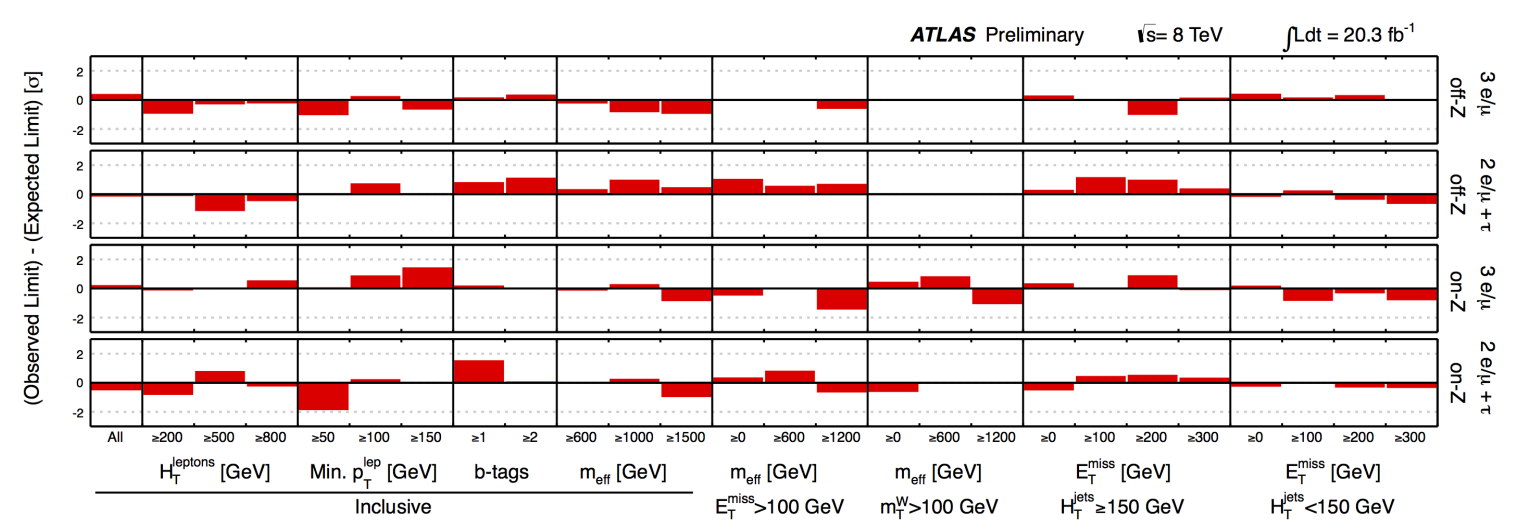

Figure 2: Deviations of observed limits from expected limits, in units of the total uncertainty on the expected limit, for all signal regions under study [3].

set at 95\% CL on the visible cross-section, using the $\mathrm{CL}_{s}$ method [15] with RooStats [16] which is presented in Figure 2 for all 94 signal regions. The visible cross-section can be converted into model specific upper cross-section limits using fiducial efficiencies $\left(\sigma_{95}=\sigma_{95}^{v i s} / \varepsilon^{f i d}\right)$. To be able to set limits on a given model of interest, this analysis first defines a fiducial volume at the particle level, and then provides per-lepton efficiencies $\left(\varepsilon^{f i d}\right)$ within that volume as a function of $p_{T}$ and $\eta$ (charge independent). To show that this is sufficient to reproduce model dependent kinematic distributions and thus limits, the parameterisation was tested for a few new physics models, such as the vector-like B quark $\left(d_{4}\right)$ model (see [17] and [3] for details).

\begin{tabular}{|c|c|c|c|c|}
\hline Channel & $\geq 3 \ell$ (off-Z) & $\geq 3 \ell$ (on-Z) & $2 \ell+\tau$ (off-Z) & $2 \ell+\tau$ (on-Z) \\
\hline Total Expected & $259 \pm 8 \pm 41$ & $3075 \pm 36 \pm 512$ & $1237 \pm 11 \pm 293$ & $17005 \pm 43 \pm 4213$ \\
\hline Observed & 280 & 3199 & 1193 & 14733 \\
\hline
\end{tabular}

Table 1: Total number of expected background events for the four signal channels with their associated statistical and systematic uncertainties, respectively. The number of observed events is also shown [3].

\section{Four Leptons}

Explaining the miniscule masses of neutrinos within the Higgs mechanism would require an extremely small Yukawa coupling. One way to provide a more natural solution is with so-called Seesaw models, which introduce heavy right-handed leptons. In the Type-III Seesaw mechanism [18, 19, 20], at least two fermionic triplets generate the neutrino masses. The lightest triplet of which introduces: $\mathrm{N}^{0}, \mathrm{~N}^{ \pm}$, with approximately degenerate masses, $m_{N}$, on the order of $100 \mathrm{GeV}$. These states couple to gauge bosons allowing for a non-negligible production cross-section at the LHC. This analysis concentrates on heavy lepton production in the four lepton decay channel: $q \bar{q} \rightarrow W^{ \pm} \rightarrow\left(N^{0}\right)\left(N^{ \pm}\right) \rightarrow\left(W^{ \pm} \ell^{\mp}\right)\left(Z\left(\ell^{+} \ell^{-}\right) \ell^{ \pm}\right)$. The dominant background to this search is diboson ZZ production, with small contributions from processes such as ZZZ and ZWW, as well as $t \bar{t} V$, single top, and $\mathrm{Z}+\mathrm{jets}$. The $\mathrm{W}+$ jets contribution was found to be negligible. 

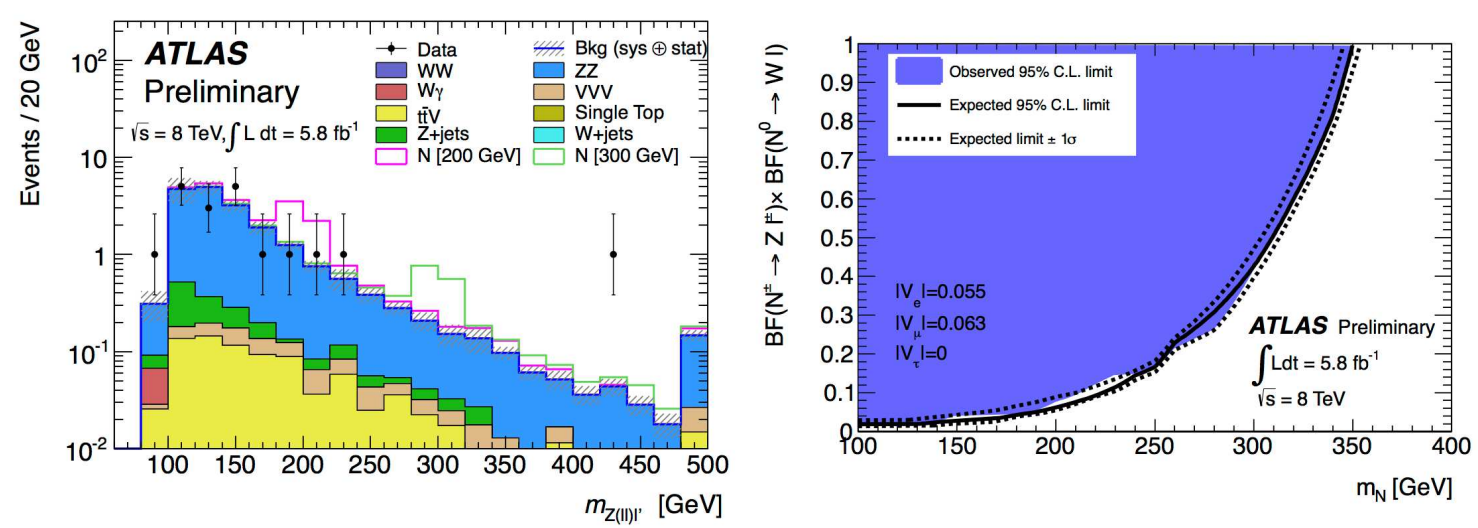

Figure 3: (left) The Invariant mass distribution of $N^{ \pm}$candidates $\left(Z_{\ell \ell} \ell_{3}\right)$ in the signal region, with the final bin including overflow events. (right) Upper exclusion limits at $95 \% \mathrm{CL}$ on $\mathscr{B}\left(N^{ \pm} \rightarrow Z \ell^{ \pm}\right) \mathscr{B}\left(N^{0} \rightarrow W^{ \pm} \ell^{\mp}\right)$ versus $m_{N}$ assuming $\left|V_{e}\right|=0.055,\left|V_{\mu}\right|=0.063$ and $\left|V_{\tau}\right|=0$ [4].

\subsection{Analysis Strategy and Selection Criteria}

For the reconstruction of heavy triplet events in this analysis, leptons are split into three categories. The first category selects Z-candidate leptons, requiring two same-flavour opposite-charge leptons consistent with having originated from a Z-boson by having $\left|m_{\ell \ell}-m_{Z}\right|<10 \mathrm{GeV}$. The second category is for a third lepton candidate which has been identified as the one closest in $\phi$ to the reconstructed $\mathrm{Z}$. The final category takes the fourth lepton to be the highest $p_{T}$ electron or muon candidate remaining in the event. Events with a second $\mathrm{Z}$ boson candidate are rejected by requiring $\left|m_{\ell_{3} \ell_{4}}-m_{Z}\right|>10 \mathrm{GeV}$, which reduces the contamination from $\mathrm{ZZ}$ events. Additionally, lepton candidates are required to pass criteria such as: a single lepton trigger, $p_{T_{\text {lead }}}>25 \mathrm{GeV}$ (all other leptons $p_{T}>10 \mathrm{GeV}$ ), $|\eta|<2.47$ (2.50) for electrons (muons), excluding the crack region, and an isolation criterion of $p_{T}^{i s o} / p_{T}<\sim 15 \%$ in a cone of $\Delta R<0.3(0.4)$. Finally, electrons are required to pass stringent identification criteria, and muons are required to have a certain number of hits in the inner detector and throughout the muon spectrometer system. This analysis uses $m_{Z_{\ell \ell} \ell_{3}}$ to reconstruct the $N^{ \pm}$invariant mass, with the presence of the fourth lepton, strongly suppressing the $\mathrm{Z}+$ jets and $\mathrm{WZ}$ background, leading to an optimal expected signal significance.

\subsection{Results}

This search finds no significant excess above the SM expectation, with one interesting $4 \mu$ event at $\sim 435 \mathrm{GeV}$. The data/MC comparison for the $m_{Z_{\ell \ell}}$ distribution is presented in Figure 3 (left). In the absence of any significant excess, 95\% CL upper limits are set on the signal production crosssection times branching fraction, using the $C L_{s}$ method with RooStats. The limit is calculated as a function of the heavy fermion mass $m_{N}$ and the product of branching fractions $\mathscr{B}\left(N^{ \pm} \rightarrow\right.$ $\left.Z \ell^{ \pm}\right) \mathscr{B}\left(N^{0} \rightarrow W^{ \pm} \ell^{\mp}\right)$, which is presented in Figure 3 (right).

\section{Conclusions}

This paper has presented three ATLAS analyses, searching for new physics in final states with multiple leptons. Each of the analyses has built upon previous work and strived to continue 
to improve the techniques and methods employed in the search and presentation of results. No significant excess of events was observed in data above the Standard Model expectation for any of the current searches. However, some of the analyses have yet to use the entire 2012 dataset, and so the possiblity of a discovery in searches using this interesting signature remain.

\section{References}

[1] ATLAS Collaboration, The ATLAS Experiment at the CERN Large Hadron Collider, 2008 JINST 3 S08003.

[2] ATLAS Collaboration, Search for excited electrons and muons with $13 \mathrm{fb}^{-1}$ of proton-proton collisions at $\sqrt{s}=8 \mathrm{TeV}$ with the ATLAS detector, ATLAS-CONF-2012-146, http://cds.cern.ch/record/1493485.

[3] ATLAS Collaboration, Search for New Phenomena in Events with Three Charged Leptons at $\sqrt{s}=$ $8 \mathrm{TeV}$ with the ATLAS detector, ATLAS-CONF-2013-070, http://cds.cern.ch/record/1562898.

[4] ATLAS Collaboration, Search for Type III Seesaw Model Heavy Fermions in Events with Four Charged Leptons using $5.8 \mathrm{fb}^{-1}$ of $\sqrt{\mathrm{s}}=8 \mathrm{TeV}$ data with the ATLAS Detector, ATLAS-CONF-2013-019, http://cds.cern.ch/record/1525526.

[5] J.C. Pati and A. Salam, Phys. Rev. D10 (1974) 275; Erratum-ibid D11 (1975) 703.

[6] U. Baur, M. Spira and P.M. Zerwas, Phys. Rev. D42 (1990) 815.

[7] K. Hagiwara, D. Zeppenfeld and S. Komamiya, Z. Phys. C29 (1985) 115.

[8] N. Cabibbo, L. Maiani and Y. Srivastava, Phys. Lett. B139 (1984) 459.

[9] ALEPH Collaboration, Phys. Lett. B385 (1996) 445; OPAL Collaboration, Eur. Phys. J. C14 (2000) 73; L3 Collaboration, Phys. Lett. B568 (2003) 23; DELPHI Collaboration, Eur. Phys. J. C46 (2006) 277.

[10] ZEUS Collaboration, Phys. Lett. B549 (2002) 32; H1 Collaboration, F.D. Aaron et al., Phys. Lett. B666 (2008) 131.

[11] CDF Collaboration, Phys. Rev. Lett. 94 (2005) 101802; CDF Collaboration, Phys. Rev. Lett. 97 (2006) 191802; D0 Collaboration, Phys. Rev. D73 (2006) 111102; D0 Collaboration, Phys. Rev. D77 (2008) 091102.

[12] ATLAS Collaboration, ATLAS-CONF-2012-008.

[13] The CMS Collaboration, Phys. Lett. B 720 (2013) 309.

[14] A. Caldwell, D. Kollar and K. Kröninger, Comput. Phys. Commun. 180 (2009) 2197.

[15] A. L. Read, Journal of Physics G: Nuclear and Particle Physics 28 (2002), no. 102693.

[16] L. Moneta et al, arXiv:1009.1003 [physics.data-an].

[17] ATLAS Collaboration, ATLAS-CONF-2013-056.

[18] R. Foot, H. Lew and X.-G. He and G.C. Joshi, Z. Phys. C 44 (1989) 441.

[19] R. Franceschini, T. Hambye, and A. Strumia, Phys.Rev. D78 (2008) 033002.

[20] F. del Aguila and J. Aguilar-Saavedra, Nucl.Phys. B813 (2009) 22-90. 